\title{
Estudio sobre Estilos de Aprendizaje mediante Minería de Datos como apoyo a la Gestión Académica en Instituciones Educativas
}

\author{
Ana Isabel Oviedo Carrascal ${ }^{1}$, Gabriel Almendrales Jiménez ${ }^{1}$ \\ ana.oviedo@upb.edu.co, a.j.gabetto@gmail.com \\ Facultad de Ingeniería en TIC, Universidad Pontificia Bolivariana, Medellín, 050004 Medellín, Colombia
}

DOI: 10.17013/risti.29.1-13

\begin{abstract}
Resumen: Los estilos de aprendizaje son las estrategias que utiliza un individuo para adquirir conocimiento, su análisis permite a las instituciones educativas mejorar los ambientes de aprendizaje, los cuales comúnmente dependen de condiciones biológicas, psicológicas, ambientales, escolares y de comunidad. En este trabajo se realiza un estudio sobre estilos de aprendizaje aplicando técnicas de minería de datos a la información derivada de la aplicación de dos test llamados VARK y CHAEA a los estudiantes de la Institución Educativa Joaquín Cárdenas Gómez del Municipio de San Carlos - Antioquia (Colombia). Como resultado de este estudio, se genera un plan de apoyo a la gestión directiva con la identificación de factores que afectan el aprendizaje. Entre los hallazgos se tiene que los estilos de aprendizaje por sí solos no influyen en la repitencia, requiere de la combinación de otros elementos como la edad y tener más de un estilo de aprendizaje.
\end{abstract}

Palabras-clave: Estilos de aprendizaje; Minería de datos; Inteligencia analítica educativa.

\section{Study on Learning Styles by Data Mining as support for Academic Management in Educational Institutions}

\begin{abstract}
Learning styles are the strategies used by a student to acquire knowledge, their analysis allows educational institutions to improve learning environments, which commonly depend on biological, psychological, environmental, school and community conditions. This paper presents a study on learning styles applying data mining techniques to the information derived from the application of two tests called VARK and CHAEA to the students of the Educational Institution "Joaquín Cárdenas Gómez" of the Municipality of San Carlos - Antioquia (Colombia). This work formulates a plan of support for the Management Directive with the list of factors that influence learning. The findings indicate that learning styles alone do not influence school failure, require the combination of other elements such as age and have more than one learning style.
\end{abstract}

Keywords: Learning styles; Data mining; educational analytic. 


\section{Introducción}

Un estilo de aprendizaje se entiende como la identificación a partir de rasgos cognitivos, afectivos y fisiológicos que manifiesta un individuo al momento de interactuar o la forma de responder al ambiente de aprendizaje (Gómez, Aduna, García, Cisneros, \& Padilla, 2004). En el contexto de la educación colombiana, la identificación de estilos de aprendizaje se orienta a las áreas de conocimiento como la psicología, pedagogía, sociología, cuyos mecanismos de validación son métodos estadísticos tanto en casos de investigaciones cuantitativas, como en los estudios centrados en la descripción de variables cualitativas (Tamayo, 1999). Las universidades en su afán de mantener su cobertura y conservar en su alma mater a sus estudiantes, han iniciado esfuerzos para profundizar en este campo y ofrecer condiciones acordes a las necesidades.

En la literatura se encuentran diversos estudios que varían en relación a las técnicas de minería de datos, a la elaboración de modelos, a la población, a las formas de adquirir los datos y a la aplicación de test de identificación de estilos de aprendizaje. Los objetivos más comunes en los estudios son el análisis de la deserción y el fracaso escolar en la formación superior mediante la aplicación de algoritmos de agrupamiento y de predicción (Puello \& Fernández, 2013) (Scheuer \& McLaren, 2012) (Vera, Morales, \& Soto, 2012) (Valía, et al., 2017) (Carmona, Vergara, Oviedo, Amon, \& Vélez, 2018).

El objeto de estudio del presente análisis es una institución educativa oficial cuyo fracaso escolar está determinado por un 14,5\%, sobre el total de 1660 estudiantes. Uno de los puntos críticos causante del fracaso escolar en la institución es el desconocimiento de los estilos de aprendizajes empleados por los estudiantes en sus actividades académicas, ocasionando el desarrollo de estrategias de enseñanza inapropiadas y conduciendo a una sucesión de consecuencias como: 1) la apatía y falta de interés por parte del educando; 2) atender inapropiadamente los problemas o barreras de aprendizaje, se registran 125 casos en el informe institucional dirigido al Departamento Administrativo Nacional de Estadística DANE (Institución Educativa Joaquín Cárdenas Gómez, 2014); 3) la repitencia de años escolares por la baja comprensión de temáticas.

Para enfrentar esta situación, en este trabajo se aplican dos instrumentos de identificación de estilos de aprendizaje, cuyos resultados se utilizan como insumo para un estudio analítico mediante diversas técnicas de minería de datos. Finalmente, los resultados del modelado analítico son utilizados para apoyar la gestión directiva en la Institución.

\section{Materiales y Métodos}

La metodología utilizada en el estudio es CRISP-DM, cuyo acrónimo en inglés significa CRoss-Industry Standard Process for Data Mining, la cual consiste en una guía de referencia de circulación libre, producto de la determinación de un consorcio de empresas europeas dedicadas al descubrimiento de conocimiento. Esta metodología es destacada por su rigurosidad y completitud a través de 6 fases: entendimiento del negocio, entendimiento de los datos, preparación de los datos, modelado, evaluación e implantación (Chapman, et al., 2000). La metodología ha sido aplicada para el análisis de datos en múltiples áreas (Oviedo \& Vélez, 2017). 


\subsection{Entendimiento del Negocio}

El presente estudio parte de adquirir la información necesaria para afrontar situaciones críticas que afectan la misión institucional la cual consiste en la formación integral de los educandos, de allí el anhelo de identificar los estilos de aprendizaje de los estudiantes para establecer su relación con el rendimiento académico e identificar otras características y comportamientos en el momento de apropiarse del conocimiento impartido. La meta es contribuir a la gestión directiva con la identificación de factores que afectan el aprendizaje de los estudiantes de la institución educativa Joaquín Cárdenas Gómez, ubicada en el Municipio de San Carlos - Antioquia, Colombia.

\subsection{Entendimiento de los Datos}

La búsqueda y recolección de los datos parte de la aplicación de dos modelos de estilos de aprendizaje: el modelo de estilos de aprendizaje de VARK denominado así por sus siglas en ingles de visual, auditivo, read/write y kinestésico es un modelo cuyas variables definen la forma de percibir el conocimiento por parte del aprendiz desde un enfoque sensorial (Fleming \& Baume, 2006).

El segundo modelo amplía un poco más la identificación de los perfiles de aprendizaje al utilizar variables orientadas a la parte aptitudinal del individuo, este modelo se conoce como CHAEA, el cual se define en cuatro categorías: activo, pragmático, teórico y reflexivo, en este concepto se identifica la postura que asume el estudiante para asimilar la información (Freibirg \& Fernández, 2013).

Adicional a los modelos de estilos de aprendizaje, se extraen las notas de los estudiantes del sistema PACSIS que opera en la institución educativa, consultando los registros de notas relacionadas a las áreas de lengua castellana, matemáticas y tecnología. Finalmente, para completar la tabla de datos los estudiantes diligenciaron un formato con preguntas personales para poder asociar patrones de comportamientos.

\subsection{Preparación de los Datos}

Esta fase consiste en aplicar controles de calidad por lo que se depuraron los datos con aplicación de técnicas estadísticas con ayuda del software WEKA y el perfilamiento de los datos con el software DQ_ANALIZER. El resultado es una tabla con 231 registros y 9 atributos. La preparación consiste en adaptar los datos para el modelado de minería, según las técnicas a desarrollar.

En el proceso de adquisición de datos sobre los estudiantes se presentaron los siguientes inconvenientes: en primer lugar, la ausencia de un sistema de registro y control de datos articulado y en segundo lugar contar con datos completos y en formato digital. Para solventar esta situación, fue necesario crear unos instrumentos de captura para recolectar los datos, se combinaron datos personales y los dos cuestionarios para identificar estilos de aprendizaje basado en dos modelos, el modelo VARK y el modelo CHAEA. A este conjunto de datos se le adicionaron los registros de notas extraídos del sistema de registro de notas de la institución.

Por inconvenientes a la hora de registrar los datos socioeconómicos, los cuales debieron ser suministrados por los acudientes, no fueron coherentes y de regular calidad a pesar de que 
el cuestionario era sencillo, pero por situaciones como el analfabetismo, núcleos familiares atípicos, la inasistencia a reuniones y falta de compromiso del acudiente, el recaudo de estos datos se volvió tedioso, por lo que se excluyeron para seguir adelante con el análisis.

\subsection{Modelamiento Analítico}

La analítica de datos permite realizar básicamente tres tipos de análisis: descriptivo, predictivo y prescriptivo. El análisis descriptivo permite descubrir conocimiento oculto en un conjunto de datos mediante agrupaciones, reglas de asociación y selección de factores. El análisis predictivo permite predecir acciones futuras mediante el análisis de un histórico de datos. Finalmente, el análisis prescriptivo permite simular escenarios para optimizar resultados. Estos análisis son desarrollados por medio de técnicas de aprendizaje de máquinas y técnicas estadísticas (Lino et al., 2017) (Oviedo, Oviedo, \& Vélez, 2015).

En este trabajo, en la fase de modelamiento se realizaron 9 experimentos, donde cada uno tiene un objetivo en particular, pero que en asocio permiten descubrir patrones de comportamientos de los estudiantes frente al aprendizaje y sus resultados académicos. Los experimentos desarrollados son de tipo descriptivo y predictivo, los cuales son:

- Análisis de perfiles de estudiantes aplicando el algoritmo K-means.

- Identificación de factores que influyen en el estilo de aprendizaje según el modelo VARK por medio de un sistema de votación de los métodos: correlaciones, árbol de decisión, regresión logística y análisis de componentes principales.

- Identificación de factores que influyen en el estilo de aprendizaje según el modelo CHAEA por medio del sistema de votación planteado anteriormente.

- Análisis de la relación entre los modelos de estilos de aprendizaje VARK y CHAEA por medio del algoritmo de reglas de asociación Apriori.

- Relación de las áreas con el modelo de aprendizaje VARK por medio del algoritmo Apriori.

- Relación de las áreas con el modelo de aprendizaje CHAEA por medio del algoritmo Apriori.

- Influencia de los estilos de aprendizaje en la repitencia por medio del algoritmo Apriori.

- Otros factores de influencia en la repitencia por medio del algoritmo de reglas de asociación Apriori.

- Predicción de riesgo de repitencia con la aplicación de un árbol de decisión.

\subsection{Evaluación}

Cada modelo es sometido a un proceso de evaluación que garantice la confiabilidad de los resultados. En los experimentos se aplican análisis descriptivos y predictivos. Los primeros son evaluados con medidas que indican la cohesión de los grupos y la confianza de las reglas de asociación. Por su parte, el modelo predictivo es evaluado según la precisión y cobertura de las predicciones.

\subsection{Implantación o Despliegue}

El estudio genera unos resultados que se convierten en indicadores para que el comité de Gestión Directiva plantee estrategias de apoyo académico, de igual forma este 
proceso motiva a profundizar en estudios analíticos y fomentar una cultura en torno a la gobernabilidad de los datos.

\section{Resultados}

A continuación, se realiza el despliegue de los resultados obtenidos de los modelos analíticos. Es un análisis desde los diversos campos de acción relacionados al proyecto iniciando por los estilos de aprendizaje, áreas académicas, repitencia y por último la gestión directiva.

\subsection{Perfilamiento de Estudiantes}

El primer experimento descubre los tipos de estudiantes de la institución educativa a partir de los estilos de aprendizaje y el desempeño académico como se presenta en la Figura 1.

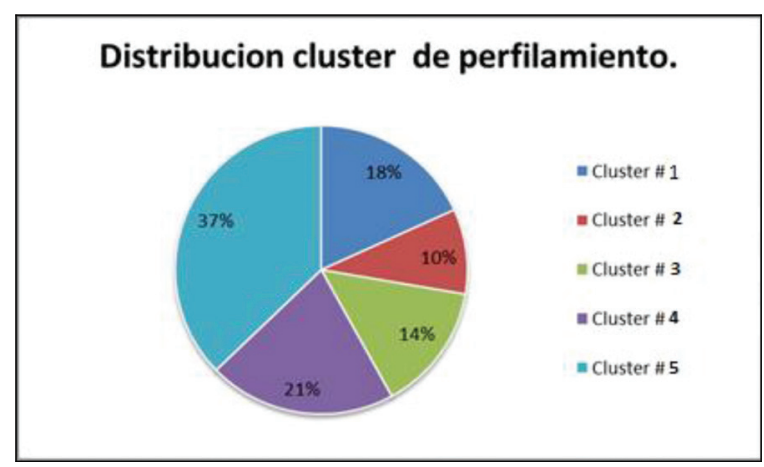

Figura 1 -Perfilamiento de los estudiantesa

El clúster \#1 presenta un porcentaje de 18\%, son estudiantes con notas de 4.0 a 4.5, con tendencias al aprendizaje reflexivo en el modelo CHAEA y en el modelo VARK el estilo es multi_estilo. Un nombre adecuado para este grupo es "Estudiantes con desempeño alto".

El clúster \#2 representado con un 10\%, los integrantes de este grupo aprenden aplicando el estilo de lectura/escritura del modelo VARK y el estilo teórico del modelo CHAEA estableciendo una relación coherente entre los dos modelos. Es el grupo que contiene mayor número de repitentes, $72 \%$ de total de integrantes dentro del grupo. Un nombre adecuado para este grupo es "Estudiantes repitentes".

El clúster \#3 comprende estudiantes de aprendizaje auditivo (VARK) y pragmático (CHAEA), en el registro de notas el rango es muy equitativo entre las tres áreas matemáticas, lengua castellana y tecnología. Este grupo puede ser llamado "Estudiantes auditivos y pragmáticos”.

El clúster \#4 son estudiantes cuyo desempeño es de nivel básico con deficiencias en las áreas matemáticas y tecnologías. Su estilo de aprendizaje en VARK es el auditivo y en el modelo CHAEA se evidencia más de un estilo de aprendizaje (Multi_estilos-CHAEA). 
El porcentaje de repitencia de este grupo es menor del 12\%. El nombre asignado a este grupo es "Estudiantes de desempeño básico".

El clúster \#5: Estudiantes con alto rendimiento los promedios de notas de las tres áreas indican que tienen un buen desempeño se caracterizan por tener un estilo kinestésico para el modelo VARK y un porcentaje de 57\% en estilo reflexivo del modelo CHAEA. Este grupo es identificado como "Estudiantes con alto desempeño".

\subsection{Factores de Influencia en Modelo de Aprendizaje VARK}

El segundo experimento está orientado a descubrir los factores que más influyen en la identificación del estilo de aprendizaje según el instrumento de VARK por medio de un sistema de votación de 4 métodos: correlaciones, árbol de decisión, regresión logística y análisis de componentes principales como se presenta en la Figura 2.

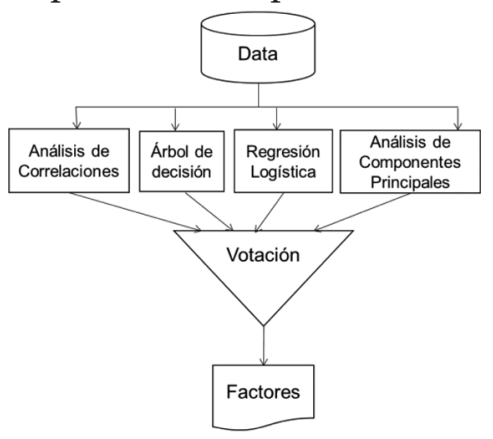

Figura 2 - Sistema de votación para descubrir los factores que influyen en el estilo de aprendizaje

El resultado encontrado indica que la nota final de matemáticas y nota final de lengua castellana son los factores más influyentes al momento de definir los estilos de aprendizaje de los estudiantes, la razón es que estas variables presentan mayor frecuencia en el conjunto de métodos aplicados, como se puede ver en la Tabla 1.

\begin{tabular}{|c|c|c|c|c|c|}
\hline Variables & $\begin{array}{l}\text { Análisis de } \\
\text { Correlación }\end{array}$ & $\begin{array}{l}\text { Árbol de } \\
\text { Decisión }\end{array}$ & $\begin{array}{l}\text { Regresión } \\
\text { Logística }\end{array}$ & $\begin{array}{l}\text { Análisis de Comp. } \\
\text { Principal }\end{array}$ & Total \\
\hline EDAD & & $\mathrm{X}$ & $\mathrm{X}$ & & 2 \\
\hline GENERO & & & $\mathrm{X}$ & $\mathrm{X}$ & 2 \\
\hline${\text { GRADO } 8^{\circ}}^{\circ}$ & & $\mathrm{X}$ & & $\mathrm{X}$ & 2 \\
\hline GRADO $9^{\circ}$ & & $\mathrm{X}$ & $\mathrm{X}$ & & 2 \\
\hline GRADO $10^{\circ}$ & & $\mathrm{X}$ & & & 1 \\
\hline GRADO $11^{\circ}$ & $\mathrm{X}$ & $\mathrm{X}$ & & & 2 \\
\hline REPITENCIA & & & & & o \\
\hline NOTA_FIN_LEN_CAST & $\mathrm{X}$ & $\mathrm{X}$ & $\mathrm{X}$ & & 3 \\
\hline NOTA_FIN_MATEMA & $\mathrm{X}$ & $\mathrm{X}$ & & $\mathrm{X}$ & 3 \\
\hline NOTA_FIN_TECNO & $\mathrm{X}$ & & & $\mathrm{X}$ & 2 \\
\hline
\end{tabular}

Tabla 1 - Consolidado de resultados experimento $\mathrm{N}^{\circ} 2$ factores que influyen en el modelo VARK 


\subsection{Factores de Influencia en Modelo de Aprendizaje CHAEA}

El tercer experimento es para identificar los factores que más influyen en la identificación del estilo de aprendizaje según el instrumento CHAEA, mediante el sistema de votación planteado en la Figura 2. En la Tabla 2 se identifican las variables de nota final de lengua castellana, nota final de matemáticas, nota final de tecnología y género como los factores que están presentes al momento de definir un estilo de aprendizaje.

\begin{tabular}{|c|c|c|c|c|c|}
\hline Variables & $\begin{array}{l}\text { Análisis de } \\
\text { Correlación }\end{array}$ & $\begin{array}{l}\text { Árbol de } \\
\text { Decisión }\end{array}$ & $\begin{array}{l}\text { Regresión } \\
\text { Logística }\end{array}$ & $\begin{array}{l}\text { Análisis } \\
\text { de Comp. } \\
\text { Principal }\end{array}$ & Total \\
\hline EDAD & & $\mathrm{X}$ & & & 1 \\
\hline GENERO & $\mathrm{X}$ & $\mathrm{X}$ & & $\mathrm{X}$ & 3 \\
\hline GRADO $8^{\circ}$ & & $\mathrm{X}$ & & $\mathrm{X}$ & 2 \\
\hline GRADO $9^{\circ}$ & & $\mathrm{X}$ & & & 1 \\
\hline GRADO $10^{\circ}$ & & $\mathrm{X}$ & $\mathrm{X}$ & & 2 \\
\hline GRADO $11^{\circ}$ & & $\mathrm{X}$ & & & 1 \\
\hline REPITENCIA & & & & & $\mathrm{O}$ \\
\hline NOTA_FIN_LEN_CAST & $\mathrm{X}$ & $\mathrm{X}$ & $\mathrm{X}$ & & 3 \\
\hline NOTA_FIN_MATEMA & $\mathrm{X}$ & & $\mathrm{X}$ & $\mathrm{X}$ & 3 \\
\hline NOTA_FIN_TECNO & $\mathrm{X}$ & & $\mathrm{X}$ & $\mathrm{X}$ & 3 \\
\hline
\end{tabular}

Tabla 2 - Consolidado de resultados experimento $\mathrm{N}^{\circ} 3$ factores que influyen en el modelo CHAEA.

\subsection{Asociaciones entre los Modelos VARK y CHAEA}

Hasta esta etapa los experimentos buscan caracterizaciones generales que permiten contextualizar los resultados obtenidos. Con este experimento se inicia una serie de indagaciones que examinan las relaciones existentes entre variables, para este caso se buscan las asociaciones entre los dos modelos de estilos de aprendizaje aplicados al estudio. El método aplicado es el Apriori arrojando como resultado que los estilos asociados directamente son auditivo (VARK) con el reflexivo (CHAEA), una segunda asociación es estilo kinestésico (VARK) con el reflexivo de (CHAEA).

\subsection{Relación de las Áreas con el Modelo de Aprendizaje VARK}

El quinto experimento muestra cómo las variables del modelo VARK se asocian con las áreas. Se parte de que las relaciones son positivas con relación al desempeño académico (ganan el curso).

Mediante la aplicación del algoritmo Apriori se encuentra que el estilo kinestésico se relaciona con las áreas de lengua castellana, matemáticas y tecnología, donde el estudiante aprende con acciones que involucran movimientos, la manipulación de objetos, dinámicas grupales y actividades donde el estudiante sea protagonista. 


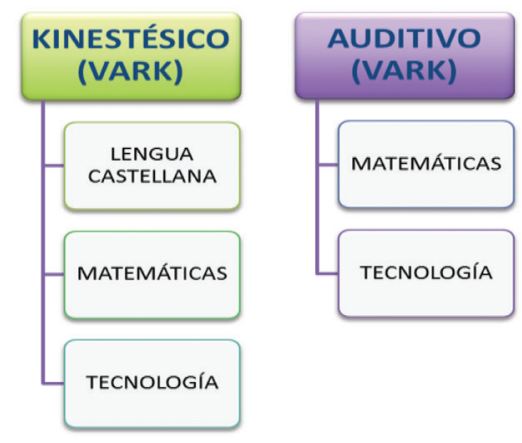

Figura 3 - Relaciones de las áreas con el modelo VARK

En la segunda asociación solo las áreas de matemáticas y tecnología comparten el estilo auditivo de VARK. En esta relación el estudiante adquiere el conocimiento por medio de instrucciones y participaciones orales y lecturas en voz alta.

\subsection{Relación de las Áreas con el Modelo de Aprendizaje CHAEA}

Para descubrir las relaciones de asocio de las áreas con el modelo de CHAEA se implementó el método Apriori. El resultado obtenido se representa en la Figura 4, donde se presentan las asociaciones que generan desempeño positivo en el rendimiento académico.

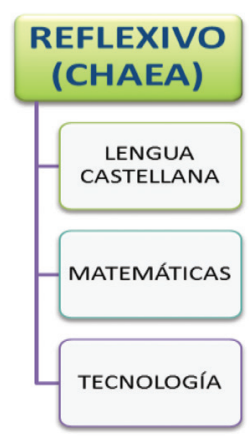

Figura 4 -Relaciones de las áreas con el modelo CHAEA

Para este caso las áreas de lengua castellana, matemáticas y tecnología el estudiante aprende reflexionando sobre el objeto de aprendizaje, requiere del espacio y tiempo.

\subsection{Influencia de los Estilos de Aprendizaje en la Repitencia}

Para conocer la influencia de los estilos de aprendizaje en la repitencia se aplicó nuevamente el método Apriori. El resultado obtenido en las reglas de asociación indica que los estilos de aprendizaje no influyen por sí solos en la repitencia. 


\subsection{Otros Factores de Influencia en la Repitencia}

Para encontrar reglas de asociación que indiquen los factores que influyen en la repitencia, se usaron variables adicionales a los estilos de aprendizaje. Con el algoritmo Apriori se encontró que el estilo reflexivo (CHAEA) en asocio de otras características como el género masculino y en edades comprendidas entre los 14 y 17 años de edad influyen para que un estudiante repita el año escolar.

Esta descripción proporciona un diagnóstico para actuar directamente sobre el grupo de estudiantes ejecutando estrategias y acciones de forma oportuna.

\subsection{Predicción de Riesgos de Repitencia}

Para precisar las características que presentan los estudiantes en riesgo de repitencia, se aplicó un método de árbol de decisión, cuyo resultado se presenta en la Figura 5. $\mathrm{Al}$ seguir las bifurcaciones decisorias se especifica los atributos que definen quienes presentan este riesgo.

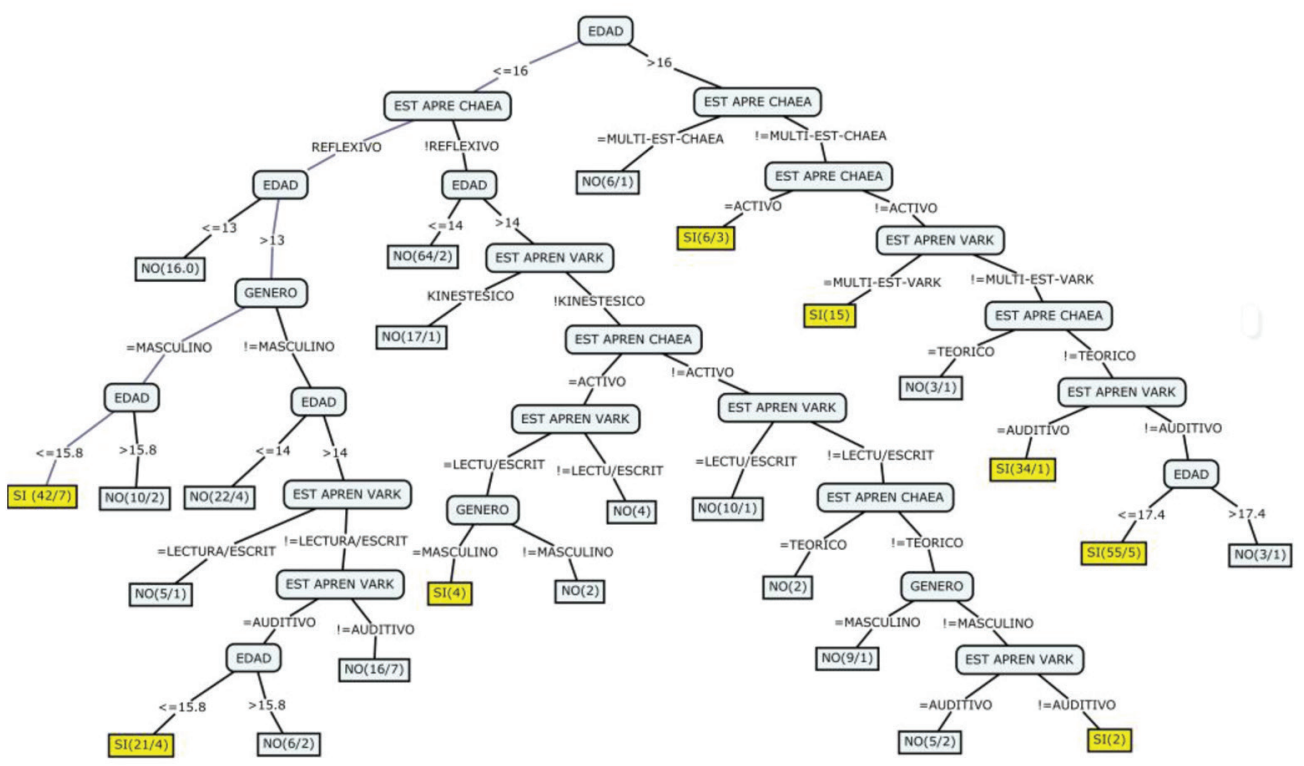

Figura 5 - Árbol de decisión para predecir riesgos de repitencia

Los estudiantes en riesgo de repitencia son los que están entre las edades igual o mayor de 14 años en ambos géneros, los estilos de aprendizaje que declaran son el reflexivo (CHAEA) combinado con el auditivo (VARK).

Un conjunto de características para identificar riesgo de repitencia es el género femenino con manifestaciones de estilos pragmático (CHAEA) y el visual (VARK), mientras otro grupo de características son el género masculino con estilos de aprendizaje activo 
(CHAEA) y lectura/escritura (VARK). Cuando la enseñanza no se acomoda a estas necesidades es cuando se activan los factores de riesgo de repitencia.

En el árbol se encuentra como riesgo de repitencia los estudiantes en edades igual o mayor de 14 años, involucra ambos géneros, de estilos de aprendizaje reflexivo (CHAEA) combinado con el auditivo (VARK).

\section{Discusión}

Se identificó una necesidad latente en la Institución Educativa Joaquín Cárdenas Gómez de carácter oficial, cuya gestión directiva plantea entre sus metas el mejoramiento de calidad educativa. Para la generación del insumo para la planeación estratégica en pro de las metas, se identificó el contexto de la organización y vocación de servicio, los cuales brindaron el soporte para generar los objetivos propios del estudio bajo la definición de la metodología CRISP-DM.

\subsection{Sobre los Estilos de Aprendizaje}

Los estilos de aprendizaje aplicados en el proyecto corresponden a dos modelos: el primero corresponde al VARK cuyos componentes aluden a los canales de percepción y recepción de la enseñanza por parte del individuo. Estos medios sensoriales son el visual, auditivo, lectura/escritura y el kinestésico. El segundo modelo por el contrario representa las aptitudes frente al aprendizaje, hace referencia al modelo CHAEA, este modelo cuenta con cuatro estilos particulares activo, reflexivo, teórico y pragmático. Para ambos modelos se adiciona una quinta variable con el objetivo de ubicar a los estudiantes que en los instrumentos aplicados tenían valores equivalentes en dos o más estilos, lo que impedía ubicarlos en uno solo, estas variables se llamaron multi_estilos_ vark y multi_estilos_chaea.

En el modelado de minería de datos se identificaron los factores que intervienen en los estilos de aprendizaje, esto permite conocer la relación de las características de los estudiantes con el comportamiento a la hora de aprender. Los factores que más influyen en el modelo de VARK son la nota final de lengua castellana y la nota final de matemáticas. Por otro lado, los factores que más influyen en el modelo CHAEA son: género, nota final lengua castellana, nota final matemáticas y nota final tecnología.

En los resultados también se descubrieron relaciones entre los dos modelos VARK y CHAEA:

El estilo auditivo de VARK se asocia directamente con el estilo reflexivo de CHAEA. La tendencia de los estudiantes al momento del aprendizaje es recibir el contenido de la enseñanza de forma auditiva, requieren escuchar las orientaciones, instrucciones y demás contenidos programados por parte del docente, al igual que necesitan un tiempo para meditar y pensar las acciones o expresiones a manifestar.

El estilo Kinestésico del modelo VARK se asocia con el estilo Reflexivo de CHAEA. En este caso el estudiante aprende directamente de la experiencia y la participación explorando el entorno con la interacción y es hábil con actividades motoras, además demuestra el desarrollo de destrezas finas o gruesas. Pero también requiere su tiempo para aprender 
cómo lo describe el aprendizaje reflexivo. En esta relación se deduce que el estudiante reflexiona sobre sus actos.

\subsection{Sobre las Áreas Académicas}

Para las áreas académicas involucradas en el estudio y la relación con los estilos de aprendizaje definidos en el modelo VARK se llega a los siguientes resultados:

El estilo dominante en los estudiantes que tiene un desempeño positivo (ganan el curso) en las áreas de matemáticas, lengua castellana y tecnología es el estilo kinestésico. Este estilo declara que el estudiante de este segmento asocia el conocimiento impartido con sensaciones y movimientos, su aprendizaje es lento, pero una vez apropiado lo conserva y profundizan, pero no compaginan muy bien cuando la información es impartida de forma visual. Esta situación repercute en el desempeño de los estudiantes porque no alcanza el nivel óptimo que es el superior, aunque su promedio de notas es de 3.23, indica un desempeño básico.

Una segunda segmentación la constituyen las áreas matemáticas y tecnología. En este par de asignaturas los estudiantes con desempeño positivo exponen un estilo de aprendizaje auditivo. Este conjunto de estudiantes, trabajan mejor cuando escuchan los contenidos o instrucciones para lograr el aprendizaje, de ahí el hecho, de que muchos susurran lo que leen para transformar la percepción lecto/escritora en auditiva.

Respecto al modelo CHAEA, se encuentra que el desempeño positivo en las áreas de matemáticas, lengua castellana y tecnología está asociado al estilo reflexivo. Este grupo de estudiantes asimilan las instrucciones y los contenidos de forma pausada, se toman el tiempo para pensar e imaginar las acciones a realizar. No se expresan con facilidad y les toma tiempo, lo que dificulta al docente percibir las necesidades de afianzar lo aprendido.

\subsection{Sobre la Repitencia}

Para prevenir la repitencia se requiere conocer las características de los estudiantes con esta problemática e identificar cuáles son las condiciones de riesgo para prevenir a futuro esta situación, si se conoce de antemano las características de dichos estudiantes, esta información se convierte en una fortaleza para la planeación estratégica de la gestión directiva.

En los resultados de este estudio se encontró que los estilos de aprendizaje por si solos NO tienen influencia en la repitencia de los estudiantes. Sin embargo, se encontró que los estilos de aprendizaje en asocio con otros factores sí influyen en dicha situación. La regla más relevante indica que el género masculino es un factor relevante, limitando a aquellos estudiantes que tiene la edad entre 14 y 17 años y específicamente los que definen un estilo de aprendizaje reflexivo según el modelo CHAEA.

Frente a esta situación es importante monitorear la relación de la edad del estudiante respecto al grado escolar, donde pueden entrar a influir elementos como el desarrollo cerebral, problemas de salud, comportamientos e intereses particulares, entre otros. Sumado a esto, si el estudiante evidencia un aprendizaje reflexivo y el docente no utiliza las técnicas de enseñanza apropiadas, no se van a obtener resultados satisfactorios. 
Iguales situaciones tienen los estudiantes que evidencian varios estilos a la hora de aprender, tanto los que demuestran ser multi_estilos_chae, como los que son multi estilo_vark. Esta situación conduce a deducir que los estudiantes no son capaces de identificar su estilo de aprendizaje, por lo cual le es difícil acomodarse a los procesos de enseñanza/aprendizaje.

\subsection{Apoyo a la Gestión Académica}

Como ente de direccionamiento, los miembros de la gestión directiva deben conocer los elementos que intervienen en la dinámica organizacional y los miembros de la comunidad a la cual prestan el servicio educativo. Por tal motivo plantean estrategias para alcanzar las metas institucionales, las cuales evaluadas anualmente contribuyen al mejoramiento institucional.

Los resultados obtenidos descubren patrones de comportamiento de los estudiantes a partir de un soporte teórico y práctico para ofrecer oportunidades de mejorar la didáctica de la enseñanza. Conocidos los estilos de aprendizaje de los estudiantes es necesario realizar ajustes en la planificación curricular (tiempo, contenidos y evaluaciones) e inclusive organizar los grupos según los patrones de comportamiento encontrados.

\section{Conclusiones}

En este trabajo se presentó un estudio sobre estilos de aprendizaje mediante minería de datos como apoyo a la gestión académica de la Institución Educativa Joaquín Cárdenas Gómez del Municipio de San Carlos (Antioquia, Colombia). Los experimentos desarrollados en el presente estudio dotan a la gestión directiva de la institución de los siguientes indicadores:

Tipos de estudiantes teniendo en cuenta el estilo de aprendizaje.

Factores que intervienen en la identificación de los estilos de aprendizaje según el cuestionario del modelo de estilos de aprendizaje de VARK y el modelo de estilos de aprendizaje de CHAEA.

Asociaciones existentes entre los dos modelos de estilos de aprendizaje aplicados al estudio. A igual que las relaciones existentes entre las áreas de conocimiento y cada uno de los modelos.

De la repitencia se identifican las características de los estudiantes con riesgo de repitencia.

Queda abierta la gama de opciones de estudios aplicando técnicas de minería de datos a nivel de las instituciones educativas de básica secundaria, debido a que la mayoría de referentes se remiten a estudios de educación superior. Para ello es necesario promover la cultura del valor de la calidad de datos en las organizaciones y su gestión para utilizar en futuros estudios.

Como trabajo futuro se propone la creación de nuevos análisis de datos de tipo prescriptivo que permitan diseñar y evaluar escenarios de acción frente a los hallazgos de este trabajo. 


\section{Referencias}

Carmona, C., Vergara, C., Oviedo, A., Amon, I., \& Vélez, G. (2018). Minería de datos y estilos de aprendizaje: una aproximación interdisciplinaria en educación superior. EnXConferencia Internacional de Ambientes Virtuales de Aprendizaje Adaptativos y Accesibles, Medellín, Colombia: Universidad Pontificia Bolivariana.

Chapman, P., Clinton, J., Kerber, R., Khabaza, T., Reinartz, T., Shearer, C., \& Wirth, R. (2000). CRISP-DM 1.o Step-by-step data mining guide. Editorial SPSS.

Institución Educativa Joaquín Cárdenas Gómez. (2014). Formulario C-60o B Formulario censal presentado al Departamento Administrativo Nacional de Estadística. Disponible en la Secretaría Académica de la Institución Educativa Joaquín Cárdenas Gómez, San Carlos, Antioquia, Colombia.

Fleming, N., \& Baume, D. (2006). Learning Styles Again: VARKing up the right tree!. Educational developments, 7(4), 4-7.

Freibirg,A., \& Fernández,M.(2013).Cuestionario Honey-Alonso deestilos deaprendizaje: Análisis de sus propiedades psicométricas en estudiantes universitarios. Summa psicológica UST, 1O(1), 103-117. DOI: 10.18774/448x.2013.10.41

Gómez, L., Aduna, A., García, E., Cisneros, A., \& Padilla, J. (2004). Manual de estilos de aprendizaje. Material Autoinstruccional Para Docentes y Orientadores Educativos (pp. 22-29). México: Secretaría de Educación Pública. Subsecretaría de Educación Media Superior. Dirección General Del Bachillerato. Dirección de Coordinación Académica.

Lino, A., Rocha, Á., \& Sizo, A. (2017). Virtual teaching and learning environments: automatic evaluation with artificial neural networks. Cluster Computing, 1-11.

Oviedo, E., Oviedo, A., \& Vélez, G. (2015). Minería de datos: aportes y tendencias en el servicio de salud de ciudades inteligentes. Revista Politécnica, 11(2O), 111-120.

Oviedo, A., \& Vélez, G. (2017). Ciencia de datos: apoyando la toma de decisiones en una ciudad inteligente. En B. Manrique (2017). Industria 4.o Escenarios e Impacto (pp. 205-218). Medellín, Colombia: Editorial Universidad de Medellín.

Puello, D. \& Fernández, M. (2013). Sistema para la detección de estilos de aprendizaje. I+D Revista de Investigaciones, 1(2), 42-49.

Scheuer, O., \& McLaren, B. (2012). Educational data mining. En Encyclopedia of the Sciences of Learning (pp. 1075-1079). Boston: Springer.

Tamayo, M. (1999). Aprender a Investigar. Serie aprender a investigar. Sta. Fe de Bogotá: ICFES.

Valía, L., Rostagno, J., Moine, J. M., Bigatti, C., Riva, F. M., \& Amar, E. (2017). Minería de datos aplicada a la educación: modelo de deserción universitaria en la Universidad Tecnológica Nacional, Facultad Regional Rosario. En XIX Workshop de Investigadores en Ciencias de la Computación. Buenos Aires, Argentina.

Vera, C., Morales, C., \& Soto, S. (2012). Predicción del fracaso escolar mediante técnicas de minería de datos. Revista Iberoamericana de Tecnologías del/da Aprendizaje/ Aprendizagem, 109. 